\title{
Schistosomiasis Protects against Multiple Sclerosis
}

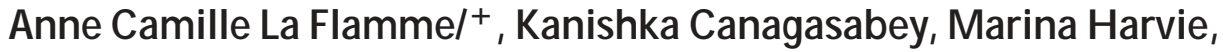 B Thomas Bäckström*}

\author{
School of Biological Sciences, Victoria University of Wellington, P.O. Box 600, Wellington, New Zealand * Malaghan Institute \\ of Medical Research, Wellington, New Zealand
}

The incidences of schistosomiasis and multiple sclerosis (MS) are mutually exclusive worldwide suggesting that schistosomiasis may offer protection against the induction of the immune-mediated disease, MS. Recent studies using the mouse model of MS, experimental autoimmune encephalomyelitis, support a direct suppression of the onset of MS by chronic Schistosoma mansoni infection. Self-reactive Th1 but not Th2 responses develop in infected mice immunized with myelin oligodendrocyte glycoprotein albeit at reduced levels indicating that the induction of auto-reactive T cells is not abolished nor phenotypically altered. CNS infiltration by inflammatory cells, particularly macrophages, is significantly reduced in $\mathrm{S}$. mansoni-infected, immunized mice compared to uninfected, immunized mice. Because activated macrophages are crucial to the induction of clinical disease, these findings support the hypothesis that differences in macrophage activation may contribute to the reduced incidence and delayed progression of experimental autoimmune encephalomyelitis during schistosomiasis.

Key words: schistosomiasis - multiple sclerosis

MULTIPLE SCLEROSIS AND EXPERIMENTAL AUTOIMMUNE ENCEPHALOMYELITIS (EAE)

Multiple sclerosis (MS) is a neurodegenerative disease characterized by progressive impairment of mobility, vision, and coordination. The course of disease is variable but can be grouped into several distinct patterns: primary progressive, secondary progressive, and relapsing-remitting (Lycklama a Nijeholt \& Barkhof 2003, Miller 2002). The immune system mediates the progression of MS, as the myelin sheaths, which insulate the nerve axons, are targeted by myelin-specific $\mathrm{T}$ cells and the resulting inflammation ultimately leads to neuronal damage (Ewing \& Bernard 1998).

EAE is the animal model commonly used to study MS (Miller \& Shevach 1998). Immunization of mice or rats with myelin proteins in the presence of a strong adjuvant leads to the development of self-reactive T cells (Kuchroo \& Weiner 1998). Although the specific myelin proteins/peptides and animal strains used can induce the full range of disease courses as found in human disease, all models share one important feature - the infiltration of autoreactive $\mathrm{T}$ cells into the central nervous system (CNS) (Kuchroo \& Weiner 1998). Type 1 CD4 T cells are essen-

Financial support: National Multiple Sclerosis Society (PP0795), the Neurological Foundation of New Zealand (0220PG), the New Zealand Lottery Health Grant (AP97228). Schistosome life cycle stages for this work were supplied through NIH-NIAID contract N01-A1-55270.

${ }^{+}$Corresponding author. Fax: +64-4-463-5331. E-mail: anne.laflamme@vuw.ac.nz.

${ }^{++}$Wellcome Trust Senior Research Fellowship in Medical Sciences, New Zealand

Received 28 May 2004

Accepted 26 July 2004 tial to the induction of this disease, however it is believed that during the chronic stages CD8 $\mathrm{T}$ cells contribute to the CNS pathology (Abdul-Majid et al. 2003). While IFN$\gamma$ does not appear to be critical for the induction of disease (Hjelmstrom et al. 1998), the IL-12p40 subunit of IL23 is essential (Cua 2003). IL-23 is believed to be central in the activation of macrophages, which are required for the initial infiltration of auto-reactive T cells into the CNS (Tran et al. 1998). In addition, macrophages mediate significant neuronal damage through the production of reactive oxygen and nitrogen intermediates and other proinflammatory mediators (Hooper et al. 1997, Kahana 2000).

Although the cause of MS is not known, both genetic and environmental factors appear to regulate disease induction (Kahana 2000). Geographically, the frequency of MS in the population is directly correlated to latitude such that countries further away from the Equator (e.g. Northern Europe, Britain, Canada, and New Zealand) have a high frequency while those in the Equatorial area (Africa, South America, and Southeast Asia) have an extremely low frequency (Kurtzke 2000). In these areas of low frequency, chronic helminth diseases such as schistosomiasis are common. Using the EAE model of MS, recent work has now shown that schistosomiasis can protect against the development of MS (La Flamme et al. 2003).

\section{SCHISTOSOMIASIS}

Schistosomiasis is caused by infection with parasitic worms of the Schistosoma species (Butterworth et al. 1994). While the worms themselves are not thought to induce significant host pathology, the deposition of eggs in the liver or bladder induces a vigorous granulomatous response (Butterworth et al. 1994). The response generated to the eggs of $S$. mansoni is characterized by high levels of Th2 cytokines (IL-4, IL-5, and IL-13) and IgE (Grzych et al. 1991). Several weeks after the onset of egg deposition and acute production of Th2 cytokines, the chronic stage begins where granulomatous pathology is down modu- 
lated and the IL-10 production is enhanced (Grzych et al. 1991). Because of the chronicity and high global prevalence of schistosomiasis, several groups have investigated the interaction of this chronic parasitic infection with other diseases both infectious and non-infectious (Cooke et al. 1999, Araujo et al. 2000, La Flamme et al. 2002, 2003, Sewell et al. 2003). In addition to preventing EAE, schistosomiasis has also been shown to inhibit the induction of other non-infectious diseases such as insulin dependent diabetes mellitus (Cooke et al. 1999). Studies are now underway to determine the mechanism behind this disease suppression.

\section{PROTECTION AGAINST THE INDUCTION OF EAE BY SCHISTOSOMIASIS}

Schistosomiasis protected against the induction of EAE by delaying the induction of disease significantly and by reducing the overall incidence of disease (Table I) (La Flamme et al. 2003). Although the onset is delayed in infected mice that developed EAE, the peak disease score is similar to uninfected mice with EAE suggesting that the protective effect of schistosomiasis may be primarily at the induction stage. In addition to live infection with $S$. mansoni, a recent study has shown that injection of a high number of the eggs alone has a similar effect on the course of EAE and that protection is only evident when the eggs are injected before disease onset (Sewell et al. 2003). Together these studies indicate that schistosomiasis protects against the induction of EAE and that egginduced responses alone can mediate this protection.

\section{REDUCTION IN SELF-REACTIVE TH1 RESPONSES}

As EAE is mediated by myelin protein-specific Th1 responses, the effect of schistosome infection on these responses was investigated. Real time PCR analysis of spinal cord transcripts and myelin peptide-specific T cell responses in the spleen showed a similar reduction in IFN- $\gamma$ and other pro-inflammatory mediators (IL-12p40, TNF- $\alpha$, and NO), but no difference in the levels of IL-10 was observed (La Flamme et al. 2003). In addition, while low levels of IL-4 were produced by antigen-stimulated splenocytes from uninfected mice with EAE, no IL-4 was produced by myelin peptide-stimulated splenocytes from S. mansoni-infected mice with EAE (La Flamme et al. 2003). Despite the absence of antigen-specific Th2 responses in infected mice with or without disease, polyclonal stimulation of splenocytes induced enhanced Th2 cytokine production compared to uninfected mice with or without EAE (Fig. 1). Interestingly, immunization with adjuvant alone appeared to enhance Th2 cytokine responses in infected mice (Fig. 1), and this increase was also evident in the serum of these mice (La Flamme et al. 2003). The detection of Th2 cytokines in the serum of infected mice suggests that this systemic Th2 environment could be mediating the protection afforded by schistosome infection. Indeed, Sewell et al. (2003) showed that the protective effect of schistosome egg injection is abolished in STAT-6-/- mice, which cannot develop Th2 responses, and this finding supports our conclusion.

\section{ALTERED CNS INFLAMMATION AND ENHANCED RE-} SOLUTION

The induction of EAE is dependent upon the infiltration of autoreactive T cells into the CNS. The delay in the onset of disease by schistosomiasis correlates to an absence of lesion development in the CNS. While the development of disease in schistosome infected mice correlates to the infiltration of inflammatory cells in the CNS, the composition of these infiltrates is significantly differ-
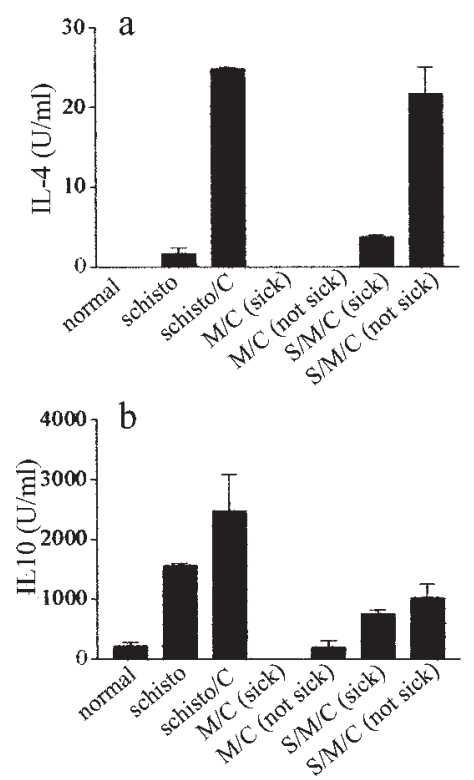

Fig. 1: schistosome-infected mice with EAE have enhanced Th2 responses compared to uninfected mice with EAE. C57BL/6 mice were infected percutaneously with 70 Schistosoma mansoni cercariae and immunized with myelin oligodendrocyte glycoprotein peptide 35-55 (MOG) in complete Freund's adjuvant (CFA) 6 weeks later as described (13). Two weeks after immunization splenocytes were isolated and stimulated with $3 \mu \mathrm{g} / \mathrm{ml}$ Con A for $72 \mathrm{~h}$. IL-4 (a) and IL-10 (b) were measured by ELISA; schisto: schistosome-infected, CFA-injected; M/C: MOG/CFA injected; SMC: schistosome infected, MOG/CFA injected. Shown are the means and SEM from 1 of 2 identical experiments.

TABLE I

Initiation and progression of experimental autoimmune encephalomyelitis is altered by schistosomiasis

\begin{tabular}{lccccc}
\hline Treatment group & Incidence $(\%)^{a}$ & $\begin{array}{c}\text { Day of disease } \\
\text { onset (avg) }\end{array}$ & $\begin{array}{c}\text { Day of peak } \\
\text { disease (avg) }\end{array}$ & Peak disease (avg) & Nr of mice \\
\hline MC & 82 & $12.3+/-0.62$ & $16.1+/-0.75$ & $3.5+/-0.4$ & 11 \\
SMC & 57 & $18.3+/-3.0^{b}$ & $23.3+/-3.9^{b}$ & $3.1+/-0.2$ & 7 \\
\hline
\end{tabular}

Shown are the results from 1 of 3 similar experiments except incidence, which has been calculated from 3 experiments; $a$ : $\mathrm{p}<0.03$, MC vs SMC incidence; paired $t$ test from 3 experiments; $b$ : $\mathrm{p}<0.03$, MC vs SMC 
ent from uninfected mice with EAE. At peak disease, the number of infiltrating macrophages is significantly reduced as detected by F4/80 immunohistochemical staining ( $\mathrm{La}$ Flamme et al. 2003) and by Mac-1 $1^{\text {high }} / \mathrm{CD} 45^{\text {high }}$ expression analysis by flow cytometry (Table II). In contrast, CD4 T cell infiltration was similar at peak disease (Table II). However, by 6 weeks after the induction of EAE, there was a marked reduction in CNS inflammation in the schistosome infected mice that developed EAE compared to uninfected mice with EAE (La Flamme et al. 2003). This reduction included CD4 T cells, CD8 T cells, macrophages, and neutrophils but not microglial cells (La Flamme et al. 2003). These results suggest that in addition to delaying the onset of disease, schistosomiasis may also accelerate the resolution of CNS inflammation.

\section{MECHANISM OF PROTECTION}

The protection afforded by schistosomiasis may be mediated by several potential mechanisms, which include but are not limited to: inhibition of autoreactive $\mathrm{T}$ cell induction, the counter-regulation of self-reactive Th1 responses by Th2 cytokines, and alteration in the cellular activation required to allow auto-reactive $T$ cells to breach the blood-brain barrier. Our results demonstrate that MOGspecific $\mathrm{T}$ cells do develop as evidenced by the production of MOG-specific IL-10 and IFN- $\gamma$ (albeit at low levels) (La Flamme et al. 2003). Because the systemic environment contains high levels of circulating Th 2 cytokines, it is possible that these cytokines may be playing a counterregulatory role in Th1 development and associated effector functions. This conclusion is strongly supported by the work of Sewell et al. (2003), who demonstrated that schistosome egg-mediated protection is dependent upon STAT-6 and thus Th2 response induction. This requirement for Th2 responses in protection against EAE cannot be directly assessed during live schistosome infection as schistosome-infected mice suffer a severe and fatal disease in the absence of Th2 responses (Brunet et al. 1997). Previous work in EAE supports the protective effect of Th2 responses and in particular IL-4 during EAE, since treatment of mice with IL-4 can significantly reduce the severity of EAE (Shaw et al. 1997). Additionally, the IL-4 expression in the CNS correlates with disease recovery and remission from relapsing episodes (Begolka et al. 1998). Moreover, IL-4 can directly reduce the ability of encephalogenic Th1 cells to induce disease (van der Veen \& Stohlman 1993).
Other immune-mediated diseases can be altered by schistosome infection. Recent work has demonstrated that the onset of diabetes in NOD mice can be inhibited by schistosome infection or the injection of schistosome eggs or antigens before the onset of disease (Cooke et al. 1999, Zaccone et al. 2003). The authors concluded that alterations in the innate immune responses after exposure to schistosome antigens led to the protection against autoimmune disease induction. Specifically, Zaconne et al. (2003) demonstrated that bone marrow dendritic cells exposed to schistosome antigens in the presence of lipopolysaccharide induced the production of IL-10 and reduced IL-12 production. In a similar manner, we have found that IFN- $\gamma$-stimulated bone marrow-derived macrophages produce more IL-10 and less IL-12p40 after stimulation with LPS in the presence of schistosome egg antigens (Fig. 2). In light of the fact that activated macrophages and IL-12p40 are essential for the induction of EAE, these results suggest that schistosomiasis may be inhibiting the onset of EAE by altering macrophage activation to reflect a more anti-inflammatory nature. Whether this protection is mediated solely by macrophages or by other cells of the innate immune system as shown by Zaccone et al. (2003) remains to be determined.
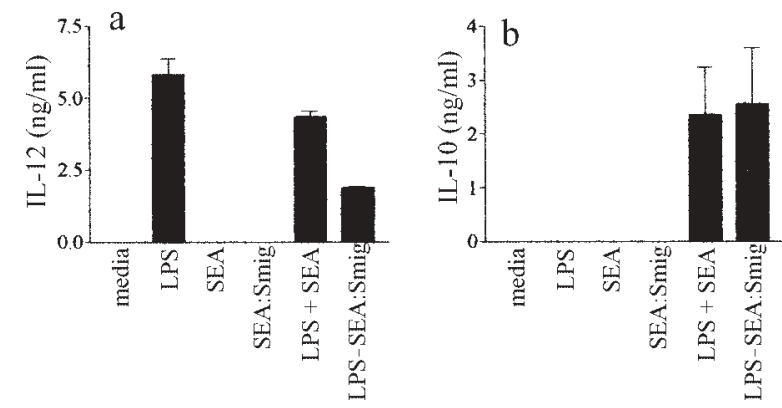

Fig. 2: schistosome products alter LPS-induced cytokine production by macrophages. Bone marrow macrophages $\left(5 \times 10^{5} / \mathrm{ml}\right)$ were stimulated with IFN- $\gamma(20 \mathrm{U} / \mathrm{ml})$ overnight and exposed to LPS $(10 \mathrm{ng} / \mathrm{ml})$, schistosome antigen $(8.5 \mu \mathrm{g} / \mathrm{ml}$; SEA), or SEA complexed with serum (1:100 dilution) from infected mice (SEA:SmIg) for 8 h. IL-12 (a) and IL-10 (b) were measured by ELISA. Shown are the means and SEM from 1 of 3 identical experiments.

TABLE II

Cell types in the spinal cord at peak disease (20 days after immunization)

\begin{tabular}{|c|c|c|c|c|c|c|c|}
\hline & $\begin{array}{c}\text { CD4+ } \\
\text { CD45+ }\end{array}$ & $\begin{array}{c}\text { CD8+ } \\
\text { CD45+ }\end{array}$ & $\begin{array}{l}\text { B220+ } \\
\text { I-Ab+ }\end{array}$ & $\begin{array}{c}\text { Mac-1+ } \\
\text { Gr-1+ }\end{array}$ & $\begin{array}{l}\text { CD45 high } \\
\text { Mac-1 + }\end{array}$ & $\begin{array}{l}\text { CD45 int. } \\
\text { Mac-1 + }\end{array}$ & $\begin{array}{l}\text { Average } \\
\text { EAE score }\end{array}$ \\
\hline Expected cell type & CD4+ T cells & CD8+ T cells & $\mathrm{B}$ cells & Neutrophils & Macrophages & Microglia & \\
\hline Normal & 0.8 & 0.4 & 0.4 & 0.8 & 1.7 & 40 & NA \\
\hline Schisto & 1.4 & 2.1 & 0.8 & 0.9 & 6.5 & 49 & NA \\
\hline MC not sick & 8.0 & 2.0 & 0.4 & 3.6 & 14 & 44 & 0 \\
\hline MC sick & 16 & 3.0 & 0.5 & 1.7 & 8.9 & 58 & 2.75 \\
\hline SMC not sick & 1.2 & 1.5 & 0.4 & 3.2 & 4.3 & 40 & 0 \\
\hline SMC sick & 15 & 1.2 & 0.4 & 3.5 & 5.4 & 62 & 2.75 \\
\hline
\end{tabular}




\section{CONCLUSION}

Current research has shown that schistosomiasis can offer protection against the development of several noninfectious immune-mediated diseases (La Flamme et al. 2003, Zaccone et al. 2003). While the precise mechanism behind this protection is not known and may differ with specific diseases, it is clear that alterations in innate immune responses and in the development or regulation of acquired immune responses play critical roles in the suppression of immune mediated diseases by schistosome infection. Furthermore, as studies have demonstrated that exposure to specific components of schistosomes (eggs and egg or worm antigens) can offer protection, it is possible that by understanding the precise mechanism by which these components inhibit disease a greater understanding of how immune-mediated diseases like MS or diabetes can be inhibited will be gained.

\section{REFERENCES}

Abdul-Majid KB, Wefer J, Stadelmann C, Stefferl A, Lassmann $\mathrm{H}$, Olsson T, Harris RA 2003. Comparing the pathogenesis of experimental autoimmune encephalomyelitis in CD4-/and CD8-/- DBA/1 mice defines qualitative roles of different T cell subsets. J Neuroimmunol 141: 10-19.

Araujo MI, Lopes AA, Medeiros M, Cruz AA, Sousa-Atta L, Sole D, Carvalho EM 2000. Inverse association between skin response to aeroallergens and Schistosoma mansoni infection. Int Arch Allergy Immunol 123: 145-148.

Begolka WS, Vanderlugt CL, Rahbe SM, Miller SD 1998. Differential expression of inflammatory cytokines parallels progression of central nervous system pathology in two clinically distinct models of multiple sclerosis. J Immunol 161: 4437-4446.

Brunet LR, Finkelman FD, Cheever AW, Kopf MA, Pearce EJ 1997. IL-4 protects against TNF-alpha-mediated cachexia and death during acute schistosomiasis. J Immunol 159: 777-785.

Butterworth AE, Curry AJ, Dunne DW, Fulford AJ, Kimani G, Kariuki HC, Klumpp R, Koech D, Mbugua G, Ouma JH 1994. Immunity and morbidity in human schistosomiasis mansoni. Trop Geogr Med 46: 197-208.

Cooke A, Tonks P, Jones, FM, O'Shea H, Hutchings P, Fulford AJ, Dunne DW 1999. Infection with Schistosoma mansoni prevents insulin dependent diabetes mellitus in non-obese diabetic mice. Parasite Immunol 21: 169-176.

Cua D, Sherlock J, Chen Y, Murphy CA, Joyce B, Seymour L, Lucian L, To W, Kwan S, Churakova T, Zurawski S, Wiekowski M, Lira SA, Gorman D, Kastelein RA, Sedgwick JD 2003. Interleukin-23 rather than interleukin-12 is the critical cytokine for autoimmune inflammmation of the brain. Nature 421: 744.

Ewing C, Bernard CC 1998. Insights into the aetiology and pathogenesis of multiple sclerosis. Immunol Cell Biol 76: 47-54.

Grzych JM, Pearce E, Cheever A, Caulada ZA, Caspar P, Heiny S, Lewis F, Sher A 1991. Egg deposition is the major stimu- lus for the production of Th2 cytokines in murine schistosomiasis mansoni. J Immunol 146: 1322-1327.

Hjelmstrom P, Juedes AE, ad Ruddle NH 1998. Cytokines and antibodies in myelin oligodendrocyte glycoprotein-induced experimental allergic encephalomyelitis. Res Immunol 149: 794-804; discussion 847-798, 855-760.

Hooper DC, Bagasra O, Marini JC, Zborek A, Ohnishi ST, Kean R, Champion JM, Sarker AB, Bobroski L, Farber JL 1997. Prevention of experimental allergic encephalomyelitis by targeting nitric oxide and peroxynitrite: implications for the treatment of multiple sclerosis. Proc Natl Acad Sci USA 94: 2528-2533.

Kahana E 2000. Epidemiologic studies of multiple sclerosis: a review. Biomed Pharmacother 54: 100-102.

Kuchroo VK, Weiner HL 1998. Antigen-driven regulation of experimental autoimmune encephalomyelitis. Res Immunol 149: 759-771; discussion 842-753, 855-760.

Kurtzke JF 2000. Multiple sclerosis in time and space-geographic clues to cause. J Neurovirol 6 (Suppl. 2): S134-140.

La Flamme AC, Ruddenklau K, Backstrom BT 2003. Schistosomiasis decreases central nervous system inflammation and alters the progression of experimental autoimmune encephalomyelitis. Infect Immun 71: 4996-5004.

La Flamme AC, Scott P, Pearce EJ 2002. Schistosomiasis delays lesion resolution during Leishmania major infection by impairing parasite killing by macrophages. Parasite Immunol 24: 339-345.

Lycklama a Nijeholt G, Barkhof F 2003. Differences between subgroups of MS: MRI findings and correlation with histopathology. J Neurol Sci 206: 173-174.

Miller DH 2002. MRI monitoring of MS in clinical trials. Clin Neurol Neurosurg 104: 236-243.

Miller SD, Shevach EM 1998. Immunoregulation of experimental autoimmune encephalomyelitis: editorial overview [editorial]. Res Immunol 149: 753-759.

Sewell D, Qing Z, Reinke E, Elliot D, Weinstock J, Sandor M, Fabry Z 2003. Immunomodulation of experimental autoimmune encephalomyelitis by helminth ova immunization. Int Immunol 15: 59-69.

Shaw MK, Lorens JB, Dhawan A, DalCanto R, Tse HY, Tran AB, Bonpane C, Eswaran SL, Brocke S, Sarvetnick N 1997. Local delivery of interleukin 4 by retrovirus-transduced $\mathrm{T}$ lymphocytes ameliorates experimental autoimmune encephalomyelitis. J Exp Med 185: 1711-1714.

Tran EH, Hoekstra K, van Rooijen N, Dijkstra CD, Owens T 1998. Immune invasion of the central nervous system parenchyma and experimental allergic encephalomyelitis, but not leukocyte extravasation from blood, are prevented in macrophage-depleted mice. J Immunol 161: 3767-3775.

van der Veen RC, Stohlman SA 1993. Encephalitogenic Th1 cells are inhibited by Th2 cells with related peptide specificity: relative roles of interleukin (IL)-4 and IL-10. $J$ Neuroimmunol 48: 213-220.

Zaccone P, Fehervari Z, Jones FM, Sidobre S, Kronenberg M, Dunne DW, Cooke A 2003. Schistosoma mansoni antigens modulate the activity of the innate immune response and prevent onset of type 1 diabetes. Eur J Immunol 33: 14391449. 